\title{
Current/Pressure Transducer Application of Model-Based Prognostics using Steady State Conditions
}

\author{
Christopher Teubert \\ SGT, Inc. \\ NASA Ames Research Center \\ Moffett Field, CA 94035 \\ 650-604-1361 \\ christopher.a.teubert@nasa.gov
}

\author{
Matthew J. Daigle \\ NASA Ames Research Center \\ Moffett Field, CA 94035 \\ matthew.j.daigle@nasa.gov
}

\begin{abstract}
Prognostics is the process of predicting a system's future states, health degradation/wear, and remaining useful life (RUL). This information plays an important role in preventing failure, reducing downtime, scheduling maintenance, and improving system utility. Prognostics relies heavily on wear estimation. In some components, the sensors used to estimate wear may not be fast enough to capture brief transient states that are indicative of wear. For this reason it is beneficial to be capable of detecting and estimating the extent of component wear using steady-state measurements. This paper details a method for estimating component wear using steady-state measurements, describes how this is used to predict future states, and presents a case study of a current/pressure (I/P) Transducer. I/P Transducer nominal and off-nominal behaviors are characterized using a physics-based model, and validated against expected and observed component behavior. This model is used to map observed steady-state responses to corresponding fault parameter values in the form of a lookup table. This method was chosen because of its fast, efficient nature, and its ability to be applied to both linear and non-linear systems. Using measurements of the steady state output, and the lookup table, wear is estimated. A regression is used to estimate the wear propagation parameter and characterize the damage progression function, which are used to predict future states and the remaining useful life of the system.
\end{abstract}

\section{Table of Contents}

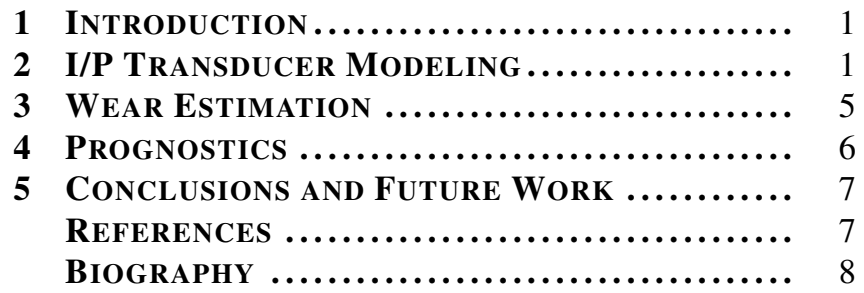

\section{INTRODUCTION}

As systems are becoming more complex, more expensive, and are being sent to increasingly unreachable places, such as space or the bottom of the ocean, wear detection, estimation, and prediction become increasingly important. Wear detection, estimation, and prediction play a critical role in preventing failure, scheduling maintenance, and improving system utility.

Many modern wear estimation techniques rely on measurement of the system's transient states [1-4]. However, in some components, the available sensors may not be fast enough to capture brief transient states that are indicative of wear.

U.S. Government work not protected by U.S. copyright.

1 IEEEAC Paper \#2323, Version Draft 1.0, Updated 15/11/2013.
This can either be a result of sensor technological limits, or budgetary constraints on sensor selection (as sensors with higher resolution and higher sampling frequencies are generally more expensive). For this reason, it is beneficial to be capable of detecting and estimating the extent of component wear as well as predicting the system's remaining useful life using only steady-state measurements. Previous work in prognostics does not address this need, and a new methodology is required.

This paper describes a method for predicting system end of life from component wear using only steady-state measurements. This is accomplished utilizing the steady-state wear estimation method described in [5]. This method utilizes a physics-based model that accounts for system behavior in both nominal and degraded conditions, and that is tuned utilizing physical specifications and knowledge of system behavior. This model is then used to map the effect of various modes of wear on steady-state behavior. Combined with regression, this method is used to predict future wear states, system remaining useful life (RUL), and system end of life (EOL) using steady-state measurements.

As a case study, this method is applied to a current/pressure transducer, henceforth referred to as an I/P Transducer or IPT. I/P Transducers are effectively pressure regulators that vary the output pressure depending on the supplied electrical current signal. They operate by throttling a nozzle to create a pressure difference across a diaphragm, which controls the throttling of a valve. These are often used for supplying precise pressures to control pneumatic actuators and valves.

The paper is organized as follows. The development of the IPT model is described in Section 2. Section 3 details the process of mapping IPT wear from steady state conditions, and using that mapping to detect and estimate wear in physical systems. The prognostic methods employed for this research are described and demonstrated in Section 4. The paper concludes with a discussion of the implications of this research and a description of future work in Section 5.

\section{I/P Transducer Modeling}

In this section, we develop a physics-based model of both healthy and faulty IPT behavior. This is used to identify how faulty behavior affects performance for development of the wear detection, estimation methodology, and prediction applications. This model was created using domain knowledge of the system's behavior and physical dimensions.

As a case study, we use a Marsh Bellofram Type 1000 IPT, illustrated in Figures 1 and 2. Some specifications for this IPT are included in Table 1 [6]. This model was chosen because 


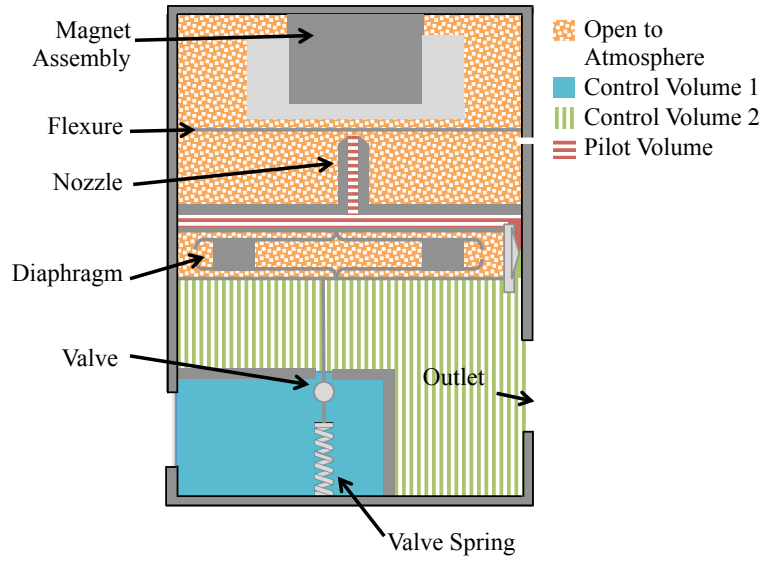

Figure 1: Current/pressure transducer schematic.

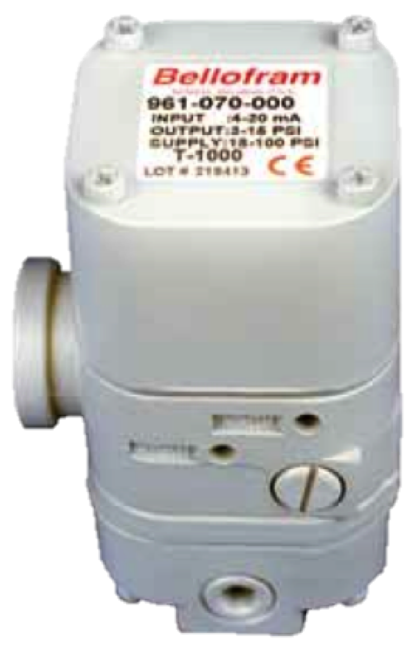

Figure 2: Current/pressure transducer.

of its use for cryogenic propellant loading applications, and, specifically in the pneumatic valve testbed at NASA Ames Research Center [7]. The IPT is divided into three distinct control volumes (CVs), each marked in a different color and pattern in the image. The IPT output pressure varies with the current supplied to the magnet assembly. When the current is high, the magnet assembly throttles the flow out of the pilot nozzle, allowing less air to escape. With a low input current more gas escapes from the nozzle, lowering the pilot pressure. The pressure difference across the diaphragm moves the valve, which adjusts the gas flow between CV1 and CV2. Adjusting this flow changes the pressure in CV2 and in the outlet.

Table 1: IPT Specifications

\begin{tabular}{ll}
\hline Name & Type 1000 IPT \\
\hline Manufacturer & Marsh Bellofram \\
\hline Supply Pressure Range & $18-100 \mathrm{psig}$ \\
\hline Input Signal Range & $4-20 \mathrm{~mA}$ \\
\hline Output Pressure Range & $3-15 \mathrm{psig}$ \\
\hline
\end{tabular}

In this section we will describe development, configuration, validation, and use of the IPT model for both the nominal and wear conditions.

\section{Problem Formulation}

We assume the system may be described by

$$
\begin{aligned}
& \dot{\mathbf{x}}(t)=\mathbf{f}(t, \mathbf{x}(t), \boldsymbol{\theta}(t), \mathbf{u}(t), \mathbf{v}(t)) \\
& \mathbf{y}(t)=\mathbf{h}(t, \mathbf{x}(t), \boldsymbol{\theta}(t), \mathbf{u}(t) \mathbf{n}(t))
\end{aligned}
$$

where $t \in \mathbb{R}$ is the continuous time variable, $\mathbf{x}(t) \in \mathbb{R}^{n_{x}}$ is the state vector, $\theta(t) \in \mathbb{R}^{n_{\theta}}$ is the parameter vector, $\mathbf{u}(t) \in \mathbb{R}^{n_{u}}$ is the input vector, $\mathbf{v}(t) \in \mathbb{R}^{n_{v}}$ is the process noise vector, $\mathbf{f}$ is the state equation, $\mathbf{y}(t) \in \mathbb{R}^{n_{y}}$ is the output vector, $\mathbf{n}(t) \in \mathbb{R}^{n_{n}}$ is the measurement noise vector, and $\mathbf{h}$ is the output equation.

In prognostics, we are interested in when the performance of a system lies outside some desired region of acceptable behavior. For this purpose, we define a threshold function $T_{E O L}(\mathbf{x}(t), \boldsymbol{\theta}(t), \mathbf{u}(t))$, such that $T_{E O L}$ evaluates to 1 when the performance is deemed unacceptable, and 0 otherwise [1].

At some point in time, $t_{P}$, the system is at $\left(\mathbf{x}\left(t_{P}\right), \boldsymbol{\theta}\left(t_{P}\right)\right)$ and we are interested in predicting the time point $t$ at which this state will evolve to $(\mathbf{x}(t), \boldsymbol{\theta}(t))$ such that $T_{E O L}(\mathbf{x}(t), \boldsymbol{\theta}(t), \mathbf{u}(t))=1$. Using $T_{E O L}$, we formally define EOL with

$$
\begin{aligned}
& E O L\left(t_{P}\right) \triangleq \\
& \quad \inf \left\{t \in \mathbb{R}: t \geq t_{P} \wedge T_{E O L}(\mathbf{x}(t), \boldsymbol{\theta}(t) \mathbf{u}(t))=1\right\},
\end{aligned}
$$

i.e., EOL is the earliest time point at which $T_{E O L}$ is met. RUL is expressed using EOL as

$$
R U L\left(t_{P}\right) \triangleq E O L\left(t_{P}\right)-t_{P} .
$$

In model-based prognostics $[1,2,4]$, the prognostics problem is composed of two distinct steps: estimation and prediction. For estimation, the objective is in general to estimate the states $\mathbf{x}(t)$, which include wear variables $\mathbf{x}_{w}$, and the wear parameters $\boldsymbol{\theta}_{w} \subseteq \boldsymbol{\theta}$. In this work, we make the single fault assumption, so only we consider only a single fault parameter at a time, and, for the fault models used in this paper, we consider only a single associated wear parameter $\theta_{w} \in \boldsymbol{\theta}_{w}$. In steady-state conditions, the states of the IPT are known except for the fault variable, so we need only estimate the fault variable and its wear parameter, using the measured steady state output, $\mathbf{y}_{\mathbf{S S}}$, and a known input, $\mathbf{u}$. Given estimates of the fault parameter and wear parameter, we can predict the growth of the fault and compute when the system will reach EOL, i.e., the time at which $T_{E O L}$ evaluates to 1 .

\section{Nominal Model}

The IPT model was developed using mass and energy balances. Each CV contains gas at a specific pressure, changing depending on the gas in-flow and out-flow. The system's state consists of the pressures at each control volume $\left(p_{1}(t), p_{2}(t), p_{\text {pilot }}(t)\right)$, the position and velocity of the valve $\left(x_{V}(t)\right.$ and $v_{V}(t)$, respectively), and the flexure position and velocity $\left(x_{F}(t)\right.$ and $v_{F}(t)$, respectively).

The IPT performance is dependent on the supply pressure provided at the inlet, $p_{i}(t)$, and the signal current sent to the magnet assembly, $i(t)$. These two values make up the input 
vector, $\mathbf{u}(t)$. For the IPT being modeled, the signal current is between 4 and $20 \mathrm{~mA}$, which varies the outlet pressure, $p_{\text {out }}(t)$, between $3-15$ psig. Outlet pressure is considered to be the only value in the output vector, $\mathbf{y}(t)$.

The input $(\mathbf{u}(t))$, state $(\mathbf{x}(t))$, state derivative $(\dot{\mathbf{x}}(t))$ and output $(\mathbf{y}(t))$ vectors are summarized below:

$$
\begin{gathered}
\mathbf{u}(t)=\left[\begin{array}{c}
p_{i}(t) \\
i(t)
\end{array}\right] \\
\mathbf{x}(t)=\left[\begin{array}{c}
p_{1}(t) \\
p_{2}(t) \\
p_{\text {pilot }}(t) \\
p_{\text {out }}(t) \\
x_{V}(t) \\
v_{V}(t) \\
x_{F}(t) \\
v_{F}(t)
\end{array}\right] \\
\dot{\mathbf{x}}(t)=\left[\begin{array}{c}
\dot{p}_{1}(t) \\
\dot{p}_{2}(t) \\
\dot{p}_{\text {pilot }}(t) \\
\dot{p}_{\text {out }}(t) \\
v_{V}(t) \\
a_{V}(t) \\
v_{F}(t) \\
a_{F}(t)
\end{array}\right] \\
\mathbf{y}(t)= \\
\\
p_{\text {out }}(t)
\end{gathered}
$$

Here velocity, $v$, and acceleration, $a$, are defined as the derivative of position, $x$, and velocity, respectively. Additionally, gas flow into a control volume from a bordering control volume is represented by $\dot{q}_{i j}$, where the subscript $i$ represents the first control volume and $j$ the bordering one and $\dot{q}_{i j}$ is the fluid flow into $i$ from $j$. The flow, $\dot{q}_{i j}$, is a function of the pressure in the control volume, $p_{i}$, pressure in the second control volume, $p_{j}$, the area of the opening between them, $A_{i j}$, and the discharge coefficient, $C_{d}$ [8]. These equations are summarized below:

$$
\begin{aligned}
\dot{x} & =v \\
\dot{v} & =a \\
\dot{q}_{i j} & =C_{d} A_{i j} C_{l} \sqrt{\left|p_{i}^{2}-p_{j}^{2}\right|} * \operatorname{sgn}\left(p_{i}^{2}-p_{j}^{2}\right) \\
C_{l} & =\sqrt{\frac{\gamma}{R * T}\left(\frac{2}{\gamma+1}\right)^{\frac{\gamma+1}{\gamma-1}}}
\end{aligned}
$$

here $\gamma$ represents the heat capacity ratio, $R$ represents the gas constant, and $T$ the temperature in that control volume. In this case the temperature is assumed to be constant throughout the IPT.

Each of the $\dot{p}$ terms are dependent on the bordering control volumes. The sum over all the interactions with a given control volume gives the total pressure flux. Accounting for all the bordering $\mathrm{CV}$ s the $\dot{p}$ equations become

$$
\begin{aligned}
\dot{p}_{1} & =\left(\dot{q}_{12}+\dot{q}_{10}\right) \frac{R * T_{1}}{V_{1}} \\
\dot{p}_{2} & =\left(\dot{q}_{21}+\dot{q}_{2 p}+\dot{q}_{2 O u t}\right) \frac{R * T_{2}}{V_{2}} \\
\dot{p}_{p} & =\left(\dot{q}_{p 2}+\dot{q}_{\text {pNozzle }}\right) \frac{R * T_{p}}{V_{p}} \\
\dot{p}_{\text {out }} & =\dot{q}_{\text {out } 2} \frac{R * T_{\text {out }}}{V_{\text {out }}}
\end{aligned}
$$

The signal current is supplied to the magnet assembly, which reacts, applying pressure on the flexure. This pressure is greater for greater signal currents. As the flexure stretches, it throttles the airflow out of the nozzle. For low input signals, the flexure flexes less, allowing more air to escape from the pilot volume, decreasing its pressure. The pilot volume is supplied from $C V_{2}$ by a small entry to the right of the diaphragm as seen in Figure 1. The net force on the flexure is the sum of the magnet assembly force $\left(F_{M a g}\right)$, the resistive force of the Flexure $\left(F_{\text {Flex }}\right)$, and friction $\left(F_{\text {Friction }}\right)$ :

$$
F_{F}=F_{\text {Mag }}+F_{\text {Flex }}+F_{\text {Friction }} \text {. }
$$

where the individual forces are

$$
\begin{aligned}
F_{\text {Mag }} & =\frac{i^{2}}{2}\left(C_{\text {mag }}-C_{\text {mag } 2} * r_{\text {mag }}\right) \\
F_{\text {Flex }} & =-k_{\text {Flex }}\left(x_{F}-x_{F 0}\right) \\
F_{\text {Friction }} & =C_{f} v_{F}
\end{aligned}
$$

Here the lumped parameters $C_{m a g}$ and $C_{m a g}$ include the gap between the coils and the metal, the area of the metal, the number of turns of the coil, and the magnetic constant. The coil resistance is represented by $r_{m a g}$. Here the value $C_{f}$ is the coefficient of friction.

The pressure difference between $C V_{2}$ and $C V_{\text {pilot }}$ produces a closing force on the valve. The lower the input signal the greater the closing force. The net force on the valve $\left(F_{V}\right)$ is the sum of the forces of the Valve Spring $\left(F_{V S}\right)$, the force created by the pressure difference across the Diaphragm $\left(F_{\text {Diaphragm }}\right)$, and the force of friction $\left(F_{\text {Friction }}\right)$. The throttling of this valve changes the flow rate between $C V_{1}$ and $C V_{2}$, affecting the output pressure $P_{\text {out }}$.

$$
F_{V}=F_{V S}+F_{\text {Diaphragm }}+F_{\text {Friction }}
$$

where the individual forces are

$$
\begin{aligned}
F_{V S} & =-k_{V} *\left(x_{V}-x_{V 0}\right) \\
F_{\text {Diaphragm }} & =\left(p_{\text {Pilot }}-p_{2}\right) * A_{D} \\
F_{\text {Friction }} & =C_{f} v_{V}
\end{aligned}
$$

where $A_{V}$ and $A_{D}$ are the areas of the valve and the diaphragm, respectively, and $k_{V}$ is the valve spring coefficient.

Each of these relationships were then converted to be in terms of acceleration using the following relationship

$$
\begin{aligned}
& a_{V}=\frac{1}{m_{V}} F_{V} \\
& a_{F}=\frac{1}{m_{F}} F_{F}
\end{aligned}
$$




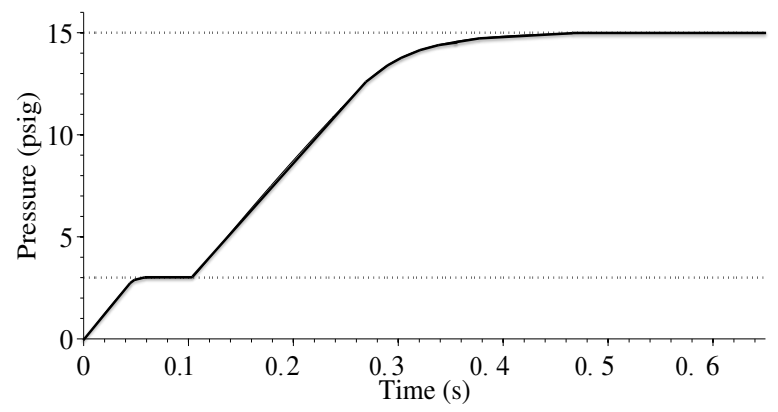

(a) Outlet Pressure, $P_{\text {out }}$

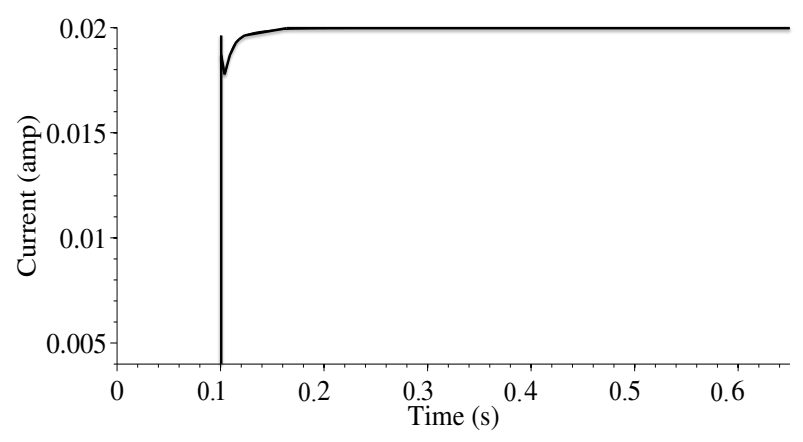

(b) Current, $i$

Figure 3: Outlet pressure for different currents.

where $m_{V}$ and $m_{F}$ are the mass of the valve and flexure, respectively.

The movement of both the flexure and the valve are derived by integrating their respective acceleration equations. The nominal output pressure response is illustrated in Figure 3a, with its respective signal current in Figure $3 \mathrm{~b}$.

This IPT model was qualitatively validated by comparing the simulated behavior with known behavior. This domain knowledge was gathered from system documentation, conversations with the manufacturing company, and observations of actual behavior.

\section{Wear Model}

Through discussions with the manufacturers and with users of I/P transducers and similar components, five possible wear modes were indicated. These wear modes are described below:

1. Inlet Leak A leak where the supply pressure is provided to $C V_{1}$. Modeled by adding a leak of area $A_{\text {in }}$ for fluid flow between $C V_{1}$ and the surrounding environment (at $1 \mathrm{~atm}$ ). The resulting fluid flow is represented by

$$
\dot{q}_{i n}=C_{d} A_{i n} C_{l} \sqrt{p_{1}^{2}-p_{a t m}^{2}}
$$

2. Valve Seat Leak A leak between $C V_{1}$ and $C V_{2}$. Modeled by adding a leak of area $A_{V S}$ for fluid flow between $C V_{1}$ and $C V_{2}$. A negative $A_{V S}$ models clogging of the valve.

$$
\dot{q}_{V S}=C_{d} A_{V S} C_{l} \sqrt{\left|p_{1}^{2}-p_{2}^{2}\right|} * \operatorname{sgn}\left(p_{1}^{2}-p_{2}^{2}\right)
$$

3. Outlet Leak A leak at the outlet. Modeled by adding a leak of area $A_{\text {out }}$ for fluid flow between $C V_{2}$ and the surrounding environment (at $1 \mathrm{~atm}$ ).

$$
\dot{q}_{\text {out }}=C_{d} A_{\text {out }} C_{l} \sqrt{p_{2}^{2}-p_{\text {atm }}^{2}}
$$

4. Valve Spring Weakening A weakening of the valve spring. Modeled by decreasing the spring coefficient, $k_{V}$.

5. Magnet Assembly Weakening A weakening of the magnet assembly. Modeled by increasing the resistance in the magnet coils, $r_{\text {mag }}$.

The wear state vector, $\mathbf{x}_{\mathbf{w}}$, is added to the state vector to model wear, and the additional equations are added to $f$. The wear state vector consists of values representing the state of wear for each of the five wear modes, is shown in the below equation

$$
\mathbf{x}_{\mathbf{w}}=\left[\begin{array}{c}
A_{\text {in }} \\
A_{V S} \\
A_{\text {out }} \\
k_{V} \\
r_{\text {mag }}
\end{array}\right]
$$

Wear by its nature progresses with time and/or use. This distinguishes it from event-driven faults, and allows for the implementation of prognostics. This model implements wear propagation models for each of the wear modes described above.

In the case of the valve spring, the wear rate, $\dot{k}(t)$, is a function of the wear rate coefficient, $w_{k}$, the force exerted by the spring, and the valve velocity [9]. Spring wear is therefore a function of use instead of time. The wear propagation equation has been included below in Equation 30.

$$
\dot{k}(t)=-w_{k}\left|F_{s}(t) v(t)\right|
$$

Leak propagation is a function of the exposed area, or the circumference of the leak, which is proportional to the square root of the area. The resulting equations for inlet leak, valve seat leak, and outlet leak are shown in Equations 3133 , where $w_{\text {leak }}$ is the leak wear rate coefficient. This relationship causes the area to change quadratically with time.

$$
\begin{aligned}
\dot{A}_{\text {in }}(t) & =w_{\text {Lin }} * \sqrt{A_{\text {in }}(t)} \\
\dot{A}_{V S}(t) & =w_{\text {LVS }} * \sqrt{A_{V S}(t)} \\
\dot{A}_{\text {out }}(t) & =w_{\text {Lout }} * \sqrt{A_{\text {out }}(t)}
\end{aligned}
$$

Coil degradation, like spring wear, is a function of use. Coil degradation is proportional to the electrical power through the coil, $P$, as seen below in Equation 34. The power is a function of the supplied current, $i$, and the operating voltage, $V$ (Equation 35).

$$
\begin{array}{r}
\dot{r}=w_{\text {Coil }} * P \\
P=i * V
\end{array}
$$

The wear parameters vector, $\boldsymbol{\theta}_{\boldsymbol{w}}$, consisting of values representing the wear parameter change rate for each of the five wear modes, is shown in the below equation

$$
\boldsymbol{\theta}_{\boldsymbol{w}}=\left[\begin{array}{c}
w_{k V} \\
w_{\text {Lin }} \\
w_{\text {Lout }} \\
w_{\text {LVS }} \\
w_{\text {rmag }}
\end{array}\right]
$$




\section{Model Verification and Validation}

To test non-damaged model realism, a series of experiments were conducted using the IPT from the Pneumatic Valve Testbed. These experiments involved taking measurements of the tuned IPT's steady state output pressure for a variety of input currents. The results showed that the output pressure is nearly linear with input current, as expected. These results were then compared with the modeled results. The model is designed so that the results measured from the IPT and the modeled results are very similar.

\section{WEAR ESTIMATION}

Wear estimation is the process of estimating the current extent of wear on a system. This is important for prognostics (predicting failure), scheduling maintenance, and triggering automated mitigation actions. This is often done using methods such as a Kalman Filter or Particle Filter [10,11]. In this work, since we have only steady-state measurements to use for prognostics, there is nothing to track since the transients are missed, and so a filtering approach would not be appropriate. Instead we employ an algorithm to directly estimate unknown parameters form steady-state measurements, given the model.

A lookup table method was used for fault estimation. This method was chosen because of its fast, efficient nature and its ability to be applied to both linear and non-linear systems. To define this lookup table the I/P transducer was simulated at various states of each wear mode and various input currents. The steady state output pressure was recorded for each case. The result was used as a reverse lookup table to estimate the wear given a specific observed steady state output pressure for a given input current. Values between data points were linearly interpolated. This was found to be sufficiently accurate given a high granularity lookup table. The granularity of the lookup table can be adjusted to increase accuracy as needed.

The resulting outlet pressure for each fault mode given a high and low input current can be seen in Figure 4. Here the outlet pressure given a high input current is indicated by the green dashed line, while that based on a low current is indicated by the blue solid line. All possible values for the IPT at a given fault level fall between these two points. In this case it was found that monitoring the steady state output pressure does not allow for the estimation of the damage state in the case of an inlet leak. This leak results in a decrease in the pressure in $C V_{1}$, which does not result in a change in the output pressure until a much larger leak (around $0.2 \mathrm{~m}^{2}$ ). For this reason the Inlet Leak case has not been included in the figures.

By contrast, the valve seat leak has a definite increasing effect on the outlet pressure (Figure 4a). This change in output pressure is a result of additional gas coming into $C V_{2}$ from $C V_{1}$ through the leak opening. For a leak of $0.3 \mathrm{~mm}^{2}$ the outlet pressure increased by 0.2577 psig for a high signal current and $0.5757 \mathrm{psig}$ for a low current.

The outlet leak also has a definite and measurable effect on the outlet pressure. As the leak grows in size, more gas escapes from $C V_{2}$, resulting in a lower outlet pressure as seen in Figure $4 \mathrm{~b}$. For a leak of $5 \mathrm{~mm}^{2}$ the outlet pressure decreases by 2.101 psig for a high signal current and 0.207 psig for a low current.
The valve spring exerts a force on the valve system countering that of the diaphragm. As the spring wears, the spring coefficient, $k$, decreases. This results in a lower counter force against the diaphragm, causing an increased output pressure as the spring coefficient decreases, as seen in Figure 4c. The effect of this is much more prominent for high input current, where the force of the diaphragm is higher. For a weakening of 34.8 to a $k$ of $1146.3 \mathrm{~N} / \mathrm{m}$ the outlet pressure changed by 0.38 psig for a high signal current and 0.08 psig for a low current.

Finally, wear in the magnet-coil assembly is simulated here by increasing the coil resistance. This, in turn, reduces the force of the magnet on the flexure proportionally with input current. The decreased force results in a greater pressure difference across the diaphragm. This closes the valve, and results in a decreased output pressure as seen in Figure 4d. This effect is much almost unseen for the low input current as a result of how the effect scales with current. For an increase of $0.1 \Omega$ to a $r_{M a g}$ of $180.1 \Omega$ the outlet pressure decreased by 0.045 psig for a high signal current and remained the same for a low current.

Each of these four wear modes resulted in a change in outlet pressure. The results for single point wear have been summarized in Table 2.

Table 2: Affect of Wear Modes on Outlet Pressure

\begin{tabular}{cc} 
Wear Mode & Effect \\
\hline \hline Inlet Leak & None \\
\hline Valve Seat Leak & Increased Outlet Pressure \\
\hline Outlet Leak & Decreased Outlet Pressure \\
\hline Worn Spring & Increased Outlet Pressure \\
\hline Work Coil & Decreased Outlet Pressure \\
\hline
\end{tabular}

Once the relationship between the fault parameter $(\theta)$, input current $(i)$, and the measured steady state output $\left(\mathbf{y}_{S S}\right)$ has been determined the resulting knowledge base can be used for wear isolation and estimation.

Two measurements with two different input current levels are required to completely isolate the fault cause. This is to differentiate between two faults that result in the same effect on output pressure. For example, if the outlet pressure is measured to be higher than it should be, that could either be indicative of a worn spring or a valve seat leak. Each of these leaks has a different relationship with input current. The second measurement allows for isolation between similar such faults. For systems with additional fault modes additional measurements may be needed to isolate between similar faults.

The following section details an example of this method.

\section{Example}

For this example let us assume we have a worn spring with a spring coefficient of $1150 \mathrm{~N} / \mathrm{m}$ (down $31.1 \mathrm{~N} / \mathrm{m}$ ).

The first measurement of steady state outlet pressure is 14.69 psig at the maximum signal current of $20 \mathrm{~mA}$. Using the reverse lookup table there are two possible options: a leak in the Outlet of $55.62 \mathrm{~mm}^{2}$, or worn spring with a spring constant of $1152.8 \mathrm{~N} / \mathrm{m}$ (down 28.3).

To definitely isolate the wear mode a second measurement is 


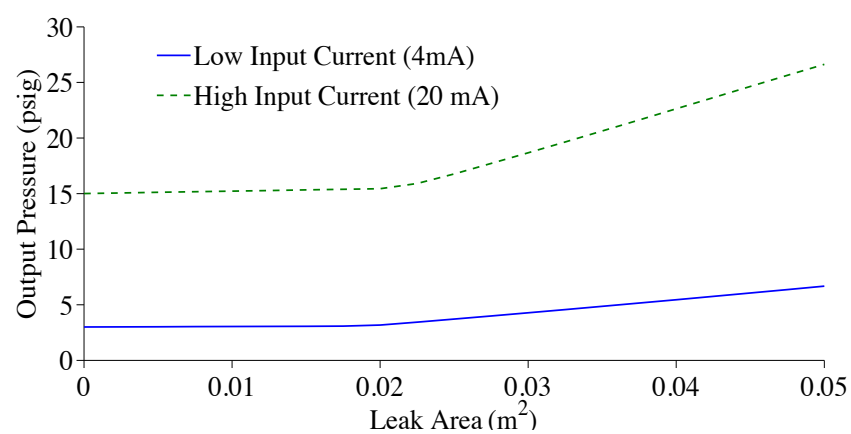

(a) Outlet Pressure for Valve Seat Leak

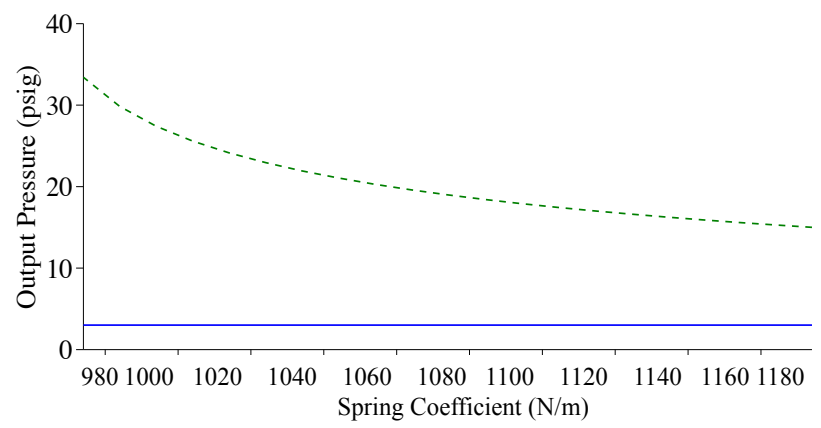

(c) Outlet Pressure for Worn Spring

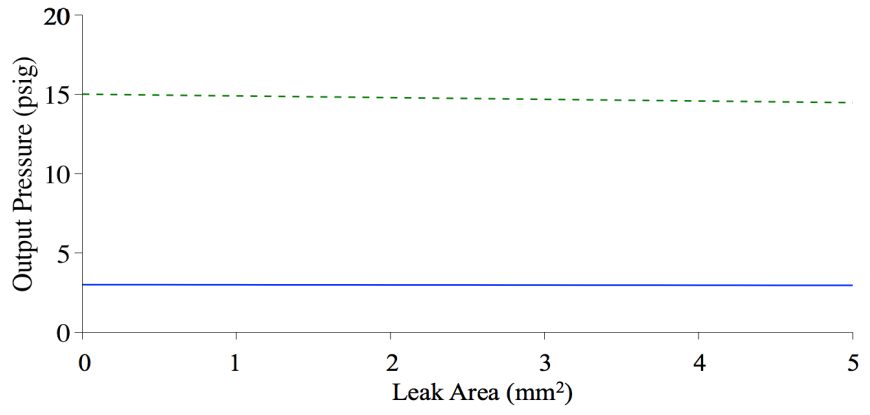

(b) Outlet Pressure for Outlet Leak

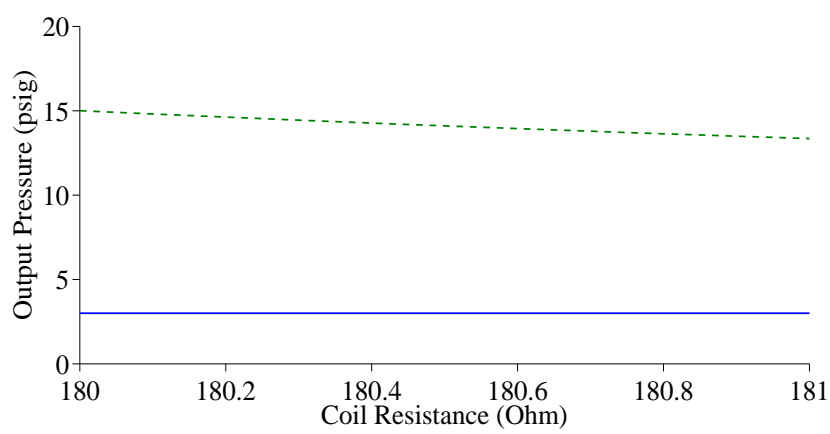

(d) Outlet Pressure for Worn Coil

Figure 4: Damaged outlet pressure.

taken, this time with the minimum signal current of $4 \mathrm{~mA}$. The outlet pressure is measured to be $2.917 \mathrm{psig}$. This can either correspond to a leak in the outlet of $12.37 \mathrm{~mm}^{2}$, or worn spring with a spring constant of $1145 \mathrm{~N} / \mathrm{m}$ (down 36.1).

Both measurements predict a worn spring of about the same wear state. This consistency leads the user to estimate that there is a worn valve spring with a spring coefficient of about $1148.9 \mathrm{~N} / \mathrm{m}$. This is calculated by taking the average of the two estimates. The difference in these measurements is due to measurement noise (n). Additional measurements at different input currents could be used to further refine the damage estimate, and filter out system noise.

\section{Prognostics}

Once wear has been discovered and diagnosed, it is useful for mitigation decisions to know how this wear will evolve over time. This information can then be used to predict the time remaining until the component is no longer usable, or the remaining useful life.

In this case wear propagation behavior is well defined. For this reason regression is used to identify the wear rate parameters, $\boldsymbol{\theta}_{\boldsymbol{w}}$ (Eq. 36), given the wear propagation equations (Eqs. 30-35). The wear knowledge base is used to determine what fault parameter value corresponds to end of life. For this case, EOL was defined as the time when the output pressure varies from the desired output pressure by $0.5 \mathrm{psig}$. Component RUL is then calculated by solving the regression equations (Eqs. 37-40) for the time that the wear will equal the end of life wear parameter, $T_{E O L}$. These equations are the solution to the wear rate differential equations (Eqs. 30$34)$. In these equations the terms $A_{i n 0}, A_{V S 0}, A_{\text {out } 0}$, and
$k_{V 0}$ represent the initial wear at time zero and $C_{f}$ is the fitting constant for each leak.

$$
\begin{aligned}
A_{\text {in }} & =C_{f i n} w_{L i n} * t^{2}+A_{\text {in } 0} \\
A_{V S} & =C_{f V S} w_{L V S} * t^{2}+A_{V S 0} \\
A_{\text {out }} & =C_{\text {fout }} w_{\text {Lout }} * t^{2}+A_{\text {out } 0} \\
k_{V} & =w_{k V} * n+k_{V 0}
\end{aligned}
$$

This method works well for the cases of the leaks, where the wear propagates quadratically with time. The spring parameter on the other hand is a function of the force on the spring and velocity of the valve (Eq. 30), and is therefore dependent on use. For this reason the regression equation is defined as a function of the number of cycles, $n$. The spring regression equation is then solved for the number of cycles at which the device will fail, $n_{E O L}$. Here a cycle is defined as a sequence where the input current is cycled between the lowest value, and the highest value, returning to the original value.

Note that for this method to work at least two wear estimations are needed, requiring at least four steady-state pressure measurements. Like with wear estimation, additional measurements improve the accuracy of the result.

The following section details an example of this method.

\section{Example}

For this example lets assume that there have been three steady state outlet pressures at three different supply currents have been recorded for each of three separate times. At each of these three times the wear estimation method described in Section 3 has been applied. The measurements and results of 
Table 3: Outlet Pressure and Wear Estimations

\begin{tabular}{lllll} 
Time (hr) & Cycle & Current & $P_{\text {out }}$ & Wear \\
\hline 0 & 0 & $20 \mathrm{~mA}$ & 14.69 & \multirow{2}{*}{ Worn Valve Spring } \\
0.1 & 0 & $4 \mathrm{~mA}$ & 2.917 & $1149 \mathrm{~N} / \mathrm{m}$ \\
0.2 & 1 & $12 \mathrm{~mA}$ & 8.804 & \\
\hline 1000 & 4322 & $20 \mathrm{~mA}$ & 14.62 & \multirow{2}{*}{1000.1} \\
4322 & $4 \mathrm{~mA}$ & 2.920 & Worn Valve Spring \\
1000.2 & 4323 & $12 \mathrm{~mA}$ & 8.770 & $1146.3 \mathrm{~N} / \mathrm{m}$ \\
\hline 2000 & 9733 & $20 \mathrm{~mA}$ & 14.58 & \multirow{2}{*}{ Worn Valve Spring } \\
2000.1 & 9733 & $4 \mathrm{~mA}$ & 2.911 & $1142.9 \mathrm{~N} / \mathrm{m}$ \\
2000.2 & 9734 & $12 \mathrm{~mA}$ & 8.747 & \\
\hline
\end{tabular}

wear estimation has been included in Table 3 . In this case the IPT monitoring system is set up to take measurements every 1000 hours for wear estimation and prediction.

given these wear parameter estimates and cycle numbers the regression algorithm reveals the following regression equation

$$
k_{V}=-.00062192 n+1148.9
$$

Using the lookup table it can then be determined that the lowest $k_{V}$ can fall without causing $P_{\text {out }}$ to change 0.5 psig at any input current is $1133.85 \mathrm{~N} / \mathrm{m}$. Solving the regression equation for $n$ predicts an end of life, $n_{E O L}$, at cycle 24360 . As the current cycle is 9734 the remaining useful life, $n_{R U L}$, is 14626 cycles. This information can then be used to schedule maintenance prior to failure. Scheduling maintenance closer to the point of failure can save both time and money.

\section{CONClusions ANd Future Work}

This paper details the development of a model-based prognostics and wear estimation approach using steady state measurements of the outlet pressure of a current/pressure transducer. This approach was then applied for the wear modes of Valve Seat Leaks, Outlet Leaks, Spring Wear, and Coil Wear, which were determined to be the most likely modes of failure.

This method was shown to be effective in identifying and predicting wear in simulations for a worn coil, worn spring, outlet leak, and leak in the valve seat. With each of these wear modes the resulting effect on the outlet pressure was different when considering two different input currents. Measuring the outlet pressure at two different input currents allows for the identification of the failure mode. The lookup table created in this study can then be used to estimate the severity of the wear. Multiple wear estimations over time can then be used to estimate the RUL. The results here demonstrate the effectiveness of steady state wear estimation for an I/P transducer.

This approach to prognostics allows for RUL estimation and wear estimation for components where sensors may not be fast enough to capture brief transient states that are indicative of wear. The results from this can be used to more efficiently schedule maintenance, or trigger automated mitigation action.

This study relied on physics-based simulations of IPT behavior validated against observations of actual system behavior. Future work includes testing this method of wear estimation in this testbed. Additionally, future work includes the estima- tion of multiple simultaneous wear modes, and uncertainty in wear estimation from steady state conditions.

\section{REFERENCES}

[1] M. Daigle and K. Goebel, "Model-based prognostics with concurrent damage progression processes," IEEE Transactions on Systems, Man, and Cybernetics: Systems, vol. 43, no. 4, pp. 535-546, May 2013.

[2] M. Orchard and G. Vachtsevanos, "A particle filtering approach for on-line fault diagnosis and failure prognosis," Transactions of the Institute of Measurement and Control, no. 3-4, pp. 221-246, Jun. 2009.

[3] B. Saha and K. Goebel, "Modeling Li-ion battery capacity depletion in a particle filtering framework," in Proceedings of the Annual Conference of the Prognostics and Health Management Society, Sep. 2009.

[4] J. Luo, K. R. Pattipati, L. Qiao, and S. Chigusa, "Modelbased prognostic techniques applied to a suspension system," IEEE Transactions on Systems, Man and Cybernetics, Part A: Systems and Humans, vol. 38, no. 5 , pp. $1156-1168$, Sep. 2008.

[5] C. Teubert and M. Daigle, "I/p transducer application of model-based wear detection and estimation using steady state conditions," in Proceedings of the Annual Conference of the Prognostics and Health Management Society, Oct. 2013, pp. 134-140.

[6] Marsh Bellofram, Type 1000 I/P \& E/P Transducers.

[7] C. Kulkarni, M. Daigle, and K. Goebel, "Implementation of prognostic methodologies to cryogenic propellant loading testbed," Proceedings of 2013 IEEE Autotestcon, September 2013.

[8] Federal Emergency Management Agency, U.S. Department of Transportation, and U.S. Environmental Protection agency, Handbook of Chemical Hazard Analysis Procedures, Appendix B, 1989.

[9] M. Daigle and K. Goebel, "A model-based prognostics approach applied to pneumatic valves," International Journal of Prognostics and Health Management, vol. 2, no. 2, Aug. 2011.

[10] M. S. Arulampalam, S. Maskell, N. Gordon, and T. Clapp, "A tutorial on particle filters for online nonlinear/non-Gaussian Bayesian tracking," IEEE Transactions on Signal Processing, vol. 50, no. 2, pp. 174-188, 2002.

[11] M. Daigle, B. Saha, and K. Goebel, "A comparison of filter-based approaches for model-based prognostics," in Proceedings of the IEEE Aerospace Conference, March 2013. 


\section{BIOGRAPHY}

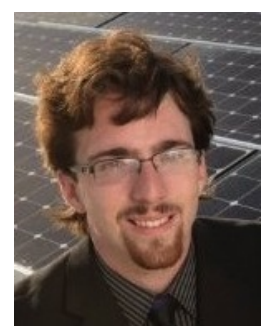

Christopher Teubert received his B.S. in Aerospace Engineering from Iowa State University in 2012. While at Iowa State University, he conducted research on asteroid deflection mission design and asteroid fragment propagation for Iowa State Universitys Asteroid Deflection Research Center (ADRC). Previous to his current position he worked as a spacecraft systems engineer for a Mars sample return mission as part of the NASA Academy Program. He is currently researching systems and algorithms for diagnostics, prognostics, and system health management for Stinger Ghaffarian Technologies, Inc. at NASA Ames Research Centers Prognostic Center of Excellence (PCoE). He plans to begin pursuing a M.S. in 2014.

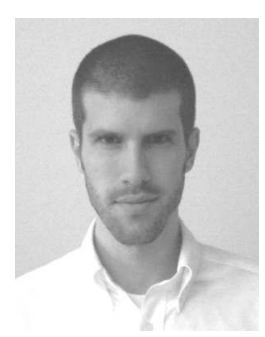

Matthew Daigle received the B.S. degree in Computer Science and Computer and Systems Engineering from Rensselaer Polytechnic Institute, Troy, NY, in 2004, and the M.S. and Ph.D. degrees in Computer Science from Vanderbilt University, Nashville, TN, in 2006 and 2008, respectively. From September 2004 to May 2008, he was a Graduate Research Assistant with the Institute for Software Integrated Systems and Department of Electrical Engineering and Computer Science, Vanderbilt University, Nashville, TN. From June 2008 to December 2011, he was an Associate Scientist with the University of California, Santa Cruz, at NASA Ames Re- search Center. Since January 2012, he has been with NASA Ames Research Center as a Research Computer Scientist. His current research interests include physics-based model-ing, model-based diagnosis and prognosis, simulation, and hybrid systems. 